\title{
Essais
}

ESSAIS

Revue interdisciplinaire d'Humanités

$8 \mid 2016$

Erreur et création

\section{Faire et refaire l'histoire de l'architecture : le rôle des archives d'architecture face aux erreurs de la recherche}

Nina Mansion

\section{(2) OpenEdition \\ Journals}

Édition électronique

URL : http://journals.openedition.org/essais/5152

DOI : 10.4000/essais.5152

ISSN : 2276-0970

Éditeur

École doctorale Montaigne Humanités

Édition imprimée

Date de publication : 15 mars 2016

Pagination : 90-106

ISBN : 978-2-9544269-7-6

ISSN : $2417-4211$

Référence électronique

Nina Mansion, «Faire et refaire l'histoire de l'architecture : le rôle des archives d'architecture face aux erreurs de la recherche », Essais [En ligne], 8 | 2016, mis en ligne le 28 octobre 2020, consulté le 30 octobre 2020. URL : http://journals.openedition.org/essais/5152 ; DOI : https://doi.org/10.4000/ essais. 5152 


\title{
Faire et refaire l'histoire de l'architecture : le rôle des archives $d^{\prime}$ architecture face aux erreurs de la recherche
}

\author{
Nina Mansion
}

"Chercher " dans le champ de l'histoire de l'architecture se révèle souvent une entreprise d'une délicatesse telle qu'elle laisse une place confortable à l'erreur. D'une part, car les obstacles et, par conséquent, les écueils potentiels de cette production complexe, collective et sociale que représente l'architecture sont nombreux. D'autre part, car l'architecture n'est pas uniquement construite mais se décline aussi au travers d'une production « conçue, imaginée, commanditée, calculée, dessinée, copiée, programmée, organisée, agencée, adaptée, rémunérée, discutée, porteuse de traditions comme de nouveautés, à la fois durable et fragile, selon les fantaisies de ses occupants et les directions prises par l'Histoire ${ }^{1} »$. S'il est aujourd'hui implicite que l'ensemble de ces interventions doit être pris en compte lors de la recherche, ce ne fut pas toujours le cas. L'histoire de l'architecture et ses méthodes d'analyse ont ainsi adopté diverses modalités de fabrication au cours du $\mathrm{XX}^{\mathrm{e}}$ siècle qui ont participé d'une construction disciplinaire cherchant à s'émanciper de sa double tutelle (histoire de l'art et recherche architecturale) pour trouver autonomie et singularité épistémologiques.

La difficulté de la recherche en histoire de l'architecture est caractérisée par deux contraintes importantes : la dispersion des sources et l'hétérogénéité des supports. Dans un éditorial de la Revue de l'art ${ }^{2}$, texte marquant la naissance d'une réflexion sur le rôle des archives en histoire de l'architecture ${ }^{3}$,

1 Conseil international des archives, Section des archives d'architecture, Manuel de traitement des archives d'architecture XIX-XX' siècles, Paris, ICA, 2000, p. 16.

2 André Chastel, "Où sont les archives de l'architecture moderne ? ", La Revue de l'art, 1975, p. 5-8.

3 Cette date de naissance doit être interrogée et soumise à la confrontation d'autres évènements et publications antérieures à la publication de l'éditorial d'André Chastel. Ainsi, la publication par Michel Le Moël d'un rapport faisant suite à la tenue du VII congrès du Conseil international des archives, sur les archives d'architecture (Michel Le Moël, «Archives de l'architecture : rapport au VII e congrès du Conseil international des archives, Moscou, 21-25 août 1972 ", 
André Chastel a été un des premiers à souligner l'éclatement des sources dans le champ de l'architecture dû, à son sens, à l'absence d'une institution nationale chargée d'encadrer la conservation et la gestion des archives d'architecture en France. La création de cette institution, qu'il avait appelée de ses vœux aux côtés d'autres acteurs de l'architecture ${ }^{4}$, ne verra le jour qu'une dizaine d'année plus tard grâce à la création du Centre d'archives d'architecture par l'Institut Français d'Architecture. Justification partielle de la dispersion, l'augmentation continuelle du volume des archives, saturant rapidement les centres de conservation, participe de l'explosion documentaire qui caractérise l'architecture 5 .

La seconde caractéristique exige du chercheur une bonne connaissance des modes de production de l'architecture et de ses acteurs, sans laquelle il est facile de passer à côté d'un pan entier du projet architectural qu'il s'agit de documenter. Ce terme de projet architectural fut consacré dans les années 1980 comme outil privilégié pour décrire, envisager et étudier les productions dans le champ de l'architecture ${ }^{6}$. Quelques précisions sur les typologies de documents qui constituent les sources de l'architecture, en tout cas du projet architectural, peuvent permettre ici de proposer une première définition de ce que l'on entend par l'expression : les " archives de l'architecture ». Ces dernières représentent l'ensemble des documents qui permettent de comprendre et plus tard, d'écrire, la vie d'un bâtiment, la biographie d'une architecture ou d'un architecte. Celle-ci est documentée par des sources qui peuvent être classées selon trois origines : les sources administratives et officielles, les documents provenant des bureaux et agences d'architectes et ceux des entrepreneurs, bureau d'études et ingénieurs ${ }^{7}$. Le premier type de source appartient aux archives dites " publiques ", tandis que les deux suivants rejoignent les archives

Moscou, URSS, 1972), remet en question le récit selon lequel Chastel aurait été le premier à évoquer ce sujet.

4 Une série d'articles et de rapports marquent l'émergence d'une réflexion unissant archives d'architecture et musée d'architecture, parmi lesquels : Bernard Huet, "Plaidoyer pour un musée d'architecture moderne ", L'architecture d'aujourd'hui, n 188, décembre 1976, p. 5 ; Bruno Foucart, "La mémoire oubliée ", L'Architecture d'Aujourd'hui, n 191, juin 1977, p. 21-23 ; Bernard Le Roy et Geneviève Ruyssen, "Les archives de l'architecture moderne: réflexions et propositions», Rapport du CERA (ENSBA), Paris, Mai-Juin 1977 ; Michel Massenet, "Rapport sur les archives de l'architecture », 1980 ; Max Querrien, "Esquisse de ce que pourrait être en France un musée de l'architecture », Rapport de mission, juin 1984.

5 Voir David Peyceré, «Les archives d'architecture entre "trop-plein” et effets de seuil ", La Revue de l'art, $\mathrm{n}^{\circ} 186,2014$, p. 17-23.

6 Voir La recherche en architecture : un bilan international - Actes du colloque Rencontres, recherche, architecture, Marseille, Nancy, Nantes, Paris, 12-13-14 juin 1984, Éditions Parenthèses, Marseille, 1986.

7 Cette classification est celle développée par le Conseil international des archives dans son Manuel de traitement des archives d'architecture XIXe-XXe siècles, édité en 2000. Conseil international des archives, Section des archives d'architecture, Manuel de traitement des archives d'architecture XIX'-XXe siècles, Paris, Conseil international des archives, 2000, $144 \mathrm{p}$. 
dites «privées " puisqu'elles proviennent d'un producteur d'origine privée. À ce propos, il ne faudra pas confondre " archives d'architectes ", d'origine uniquement privée, et " archives d'architecture " ou "documents d'architecture " qui embrassent la production dans son ensemble.

Bien qu'en histoire de l'architecture, l'ensemble de ces caractéristiques soit fondamental à considérer dans le cadre d'une recherche exigeante, l'histoire a montré que cette rigueur, si tant est qu'elle fut connue et appliquée comme telle, ne fut pas toujours respectée. La complexité de la recherche, ici rapidement défrichée, invite donc à s’interroger sur les démarches adoptées par les historiens de l'architecture pendant le $\mathrm{XX}^{\mathrm{e}}$ siècle, période qui correspond à notre champ de recherche, et par là, sur les erreurs méthodologiques qui ont pu se manifester.

\section{Erreur et histoire de l'architecture}

Invoquer l'erreur dans le champ de l'histoire de l'architecture revient à la considérer comme une faiblesse méthodologique, plutôt que comme l'exposition d'une information qui ne serait pas conforme à la vérité. Or, l'erreur n'est pas ici entendue comme une faute, ni comme l'acte de se tromper, action qui serait imputable à une singularité consciente mais comme un fait collectif, un comportement généralisé et peut-être involontaire, une de ces postures qui appartient à l'" inconscient d'école " de Pierre Bourdieu. L'erreur est souvent associée, par jeu d'association, à la fraude et à la controverse. Si l'on évacue dès maintenant la fraude qui, en ce qui nous concerne, ne correspond qu'à une réalité très marginale, la controverse, quant à elle, n'est pas étrangère au champ de l'histoire de l'architecture qui, notamment au $\mathrm{XX}^{\mathrm{e}}$ siècle, a largement reposé sur l'alimentation des polémiques et du débat autour du Mouvement moderne. En considérant l'association " erreur » et " histoire de l'architecture ", l'instinct pousserait à évoquer premièrement les erreurs, souvent anecdotiques, voire participant à des mythes, qui ont pu marquer l'art de la construction, comme l'a déjà fait Antoine Vigne ${ }^{8}$. Plus récemment et plus directement lié à la question des archives, on peut penser aux erreurs, en tout cas, aux regrets exprimés par certains historiens à propos d'une série d'interventions urbaines qui ont marqué certaines villes comme Paris au début des années 1970. Parallèlement aux luttes urbaines en Belgique qui se développent en réponse à la "bruxellisation » de la ville ${ }^{9}$, la démolition des pavillons de Baltard ${ }^{10}$, qui a suscité de vives réactions parmi les professionnels

8 Antoine Vigne, Les erreurs dans l'architecture, Paris, Éditions Courtes et longues, 2013.

9 La «bruxellisation » est un épisode de l'aménagement urbain de la capitale belge durant lequel les autorités publiques ont procédé à des vagues de démolition dans les quartiers populaires de la ville.

10 Les pavillons des Halles de Paris, conçus par l'architecte Victor Baltard, ont été détruits entre 1971 et 1973. 
et les "amoureux de Paris ", a légitimé le mouvement lancé par André Chastel appelant à la conservation des traces et des témoignages de l'architecture qui disparaît mais aussi de celle qui demeure ${ }^{11}$. Ces interventions violentes dans la ville ont été considérées par certains des acteurs du champ de l'architecture et de la protection du patrimoine comme des erreurs, des gestes immuables et indélébiles, même si ces dernières apparaissent plus aujourd'hui comme un fait sociétal qu'historique, voire historiographique. Ces personnalités furent parmi les premières à agir pour la création de centres de conservation des archives de l'architecture, comme le fit Maurice Culot ${ }^{12}$ en 1968 avec les Archives de l'architecture moderne de Belgique ou Phyllis Lambert qui, parallèlement à la constitution d'une collection de dessins d'architecture iconiques et internationaux, se mit à rassembler des fonds d'archives montréalais pour protéger le patrimoine d'une ville qu'elle voyait disparaitre ${ }^{13}$.

Si pour certains ces anecdotes de l'histoire de la conservation du patrimoine peuvent constituer les premières erreurs de l'histoire de l'architecture, les plus évidentes, notre intérêt porte sur une autre définition de la discipline, non plus envisagée comme simple champ de l'histoire, mais comme modalité d'écriture, méthode d'analyse, manière de "faire récit ». Par-là, la réflexion s'inquiète des erreurs qui ont ponctué, infiltré le champ méthodologique. Ces dernières, qui peuvent être envisagées sous forme de typologie, voient la rencontre des méthodes de la recherche scientifique et du champ de l'histoire qui nous intéresse, celui de l'histoire du patrimoine construit. Selon Jean-François Bach ${ }^{14}$, la science dite " exacte ", par opposition aux sciences " humaines " et "sociales ", reconnaît principalement trois types d'erreurs qu'il est ici possible d'utiliser pour dégager les modalités de l'erreur en histoire de l'architecture. Le premier correspond à l'erreur expérimentale qui ne connaît pas de réalité dans le champ historique où la manipulation n'apporte rien à l'analyse. Le second, en revanche, a tout à voir avec l'histoire de l'architecture puisqu'il s'agit de l'erreur d'interprétation qui est la modalité d'analyse privilégiée de l'histoire de l'art, discipline tutélaire de l'histoire de l'architecture qui n'en est actuellement qu'une spécialité des études universitaires. L'erreur d'interprétation consiste à considérer les mauvaises preuves ou à

11 Chastel avait publié à ce sujet une série d'articles dans Le Monde relatifs à la destruction ou la détérioration du patrimoine, par les guerres ou les aménagements, parmi lesquels "Les monuments détruits », 22 janvier 1946, "Comment on défigure un quartier ancien », 9 novembre 1949, «Les pierres et les hommes », 7 février 1964.

12 Maurice Culot crée et dirige les Archives d'architecture moderne de Bruxelles en 1968, avant de s'occuper du département Archives de l'IFA, créé en 1980 et d'y encadrer la création du Centre d'archives d'architecture ouvert en 1988.

13 Phyllis Lambert a crée le Centre Canadien d'Architecture en 1979.

14 Jean-François Bach, "L'erreur scientifique ", Séance solennelle de l'Académie des sciences, 21 juin 2011, Institut de France, Académie des sciences (consultation en ligne le 3 janvier 2015 : http://www.academie-sciences.fr/academie/membre/s210611_bach.pdf). 
utiliser abusivement certains éléments dans la vérification d'un postulat. Cette erreur, à l'instar du type suivant de l'erreur par ignorance, peut être inhérente au défaut de consultation des sources primaires qui a longtemps dominé une recherche faite avec peu de moyens. L'erreur par ignorance est protéiforme et repose principalement sur un manque de sources ou une méconnaissance de l'état de l'art d'un sujet de recherche. Spécifique au champ scientifique de l'histoire en tant qu'étude des évènements du passé fussent-ils politiques, économiques, artistiques ou architecturaux, le dernier type d'écart méthodologique correspond à l'erreur de datation. Plus souvent repérée dans le champ de l'architecture antique ${ }^{15}$, de l'archéologie du bâti médiéval ou dans celui de l'architecture rurale ${ }^{16}$, l'erreur de datation se caractérise soit par le choix erroné d'une date pour une autre, soit par l'utilisation d'une date symbolique pour évoquer conjointement les différentes étapes de la réalisation d'un bâtiment (conception, construction, achèvement...). Cette erreur peut s'accompagner de l'erreur de répétition, dont nous reparlerons, qui consiste à conserver une date donnée par un confrère ou un prédécesseur dont on ne sait pas nécessairement s'il la considère comme la date de conception ou d'achèvement. Afin d'éviter ce type d'erreur, on peut trouver en tête d'ouvrage une note aux lecteurs, comme le fait François Loyer dans son Histoire de l'architecture française, qui explique ne retenir que la " date du projet ${ }^{17}$. Il précise qu'il " ajoute la date d'achèvement lorsqu' elle est connue " et que " sauf exception, le début des travaux n'est donc jamais signalé ${ }^{18}$. Enfin, il existe également quelques exemples de manipulation de la datation d'un bâtiment par l'architecte, notamment lorsque ce dernier souhaite inscrire son travail dans une démarche d'innovation, conceptuelle ou technique, dont il se réclamerait le précurseur. C'est le cas de l'immeuble de la rue Franklin, conçu par les Frères Perret pour lequel les dates furent manipulées en sorte que l'immeuble apparaisse comme la première réalisation en béton armé apparent ${ }^{19}$. Ces quelques types d'erreur restent cependant mineurs en histoire de l'architecture, loin derrière des formes certes insidieuses, parfois imperceptibles aux yeux novices, mais néanmoins courantes, voire même ordinaires, en tout cas coutumières.

15 Nicolas Kyriakidis, "Erreurs à Delphes. La tholos de Marmaria au fil des interprétations ( $c a$ 1840-1940)", Anabases, 11 - 2010, p. 149-163.

16 Jean-Michel Sauget, "Datation et typochronologie de l'habitat rural de la plaine languedocienne : pièges chronologiques et familles recomposées ", In Situ, n 9, 2008, mis en ligne le 18 avril 2012, consulté le 21 novembre 2014, http://insitu.revues.org/3895.

17 François Loyer, Histoire de l'architecture française, Éditions du Patrimoine, Paris, Mengès, 2014, p. 1.

18 Ibid.

19 Cette manipulation des dates a été mise à jour et communiquée lors de séminaires organisés au début des années 2000 à l'ENSAP de Bordeaux par Gilles Ragot, mais n’a pas fait l'objet publication. 


\section{Le conflit autour de la modernité - l'erreur comme outil de construction disciplinaire}

Revenons, en premier lieu, sur l'erreur dite de répétition qui consiste à reprendre l'information sans opérer la vérification intermédiaire, à la fois interrogation de la rigueur méthodologique de la démonstration - si tant est qu'il s'agisse de démontrer une information sinon de la diffuser - et appréciation critique des éléments et sources mobilisés. Ce type d'erreur, aussi audacieux que le terme puisse paraitre, fut récurrent pendant quelques longues décennies du XXe siècle marquées par le récit du Mouvement moderne triomphant. Ce courant architectural ayant largement dominé le $\mathrm{XX}^{\mathrm{e}}$ siècle, il s'est longtemps agi d'en écrire les rebonds et sursauts, d'en entretenir la vigueur en procédant à une histoire de la nouveauté dont la méthode reposait sur la consultation privilégiée des revues d'architecture qui diffusaient et rendaient accessible l'actualité de l'architecture. Cette méthode, qui consistait à écrire les histoires de l'architecture d'après les précédentes, a bénéficié du récit fait par Chastel de la situation critique dans laquelle se trouvaient les archives d'architecture. Quand l'histoire se faisait à partir des sources primaires, c'est parce que l'historien parvenait à accéder à des fonds restés vierges de toute intervention grâce aux héritiers des architectes ou à quelques rares institutions, archives publiques ou sociétés d'architectes ${ }^{20}$, qui avaient effectué un travail de collecte. Cette histoire de l'architecture portant haut les principes du Mouvement moderne, du " modernisme » ou de la modernité en architecture selon les écoles, a aussi fait l'erreur, comme on a souvent pu lui reprocher, de n'être justement que d'actualité car sa portée historique a pris fin avec le projet qu'elle défendait ${ }^{21}$. Elle fut le fait de ceux que l'on associe désormais à la première génération d'historiens de l'architecture du $\mathrm{XX}^{\mathrm{e}}$ siècle, qui ont écrit alors que le Mouvement moderne ouvrait la voie de l'architecture, dont Nikolaus Pevsner, Sigfried Giedion ou Bruno Zevi. Andrew Leach, dans son récent ouvrage de synthèse What is architectural history?22, invite à considérer ces derniers comme les narrateurs d'une histoire qu'ils ont eux-mêmes

20 Avant la création du Centre d'archives d'architecture en 1980, la Société des architectes de Lyon, crée en 1830 avait commencé à collecter les fonds de ses membres, tout comme l'Académie d'architecture de Paris, dont la centaine de fonds complète aujourd'hui la collection du Centre d'archives d'architecture du XXe siècle, a été crée en 1840. Par ailleurs, quelques fonds étaient conservés aux Archives Nationales et une importante collection de fonds concernant l'architecture du béton armé avait été fondée par Jean-Baptiste Ache et Henri Poupée au Conservatoire National des Arts et Métiers.

21 Nous pourrions développer ce point ici en évoquant les historiens militants politiques et notamment toute une génération d'historiens communistes qui privilégient une approche progressiste de l'histoire du Mouvement moderne en négligeant plus ou moins volontairement d'autres aspects qui ne serviraient pas leur discours.

22 Andrew Leach, What is architectural history?, Cambridge, Malden, Polity, 2010, p. 77. 
vécus, observateurs privilégiés mais aussi acteurs impliqués. Déjà avant lui, Panayotis Tournikiotis, avait porté l'attention sur l'importance des textes et des modalités d'écriture de l'histoire de l'architecture, et notamment de celle du Mouvement moderne dans sa célèbre thèse Historiography of Modern Architecture $^{23}$. Dans le sillage de Tournikiotis, Leach reprend en effet le concept de généalogie $e^{24}$, qu'il transforme en génération, pour mettre en évidence les différentes démarches ${ }^{25}$ des historiens selon leur époque et leur relation à l'architecture dont ils essayaient de faire l'histoire. Pour Leach, les auteurs de la première génération (Pesvner, Giedion, Zevi) peuvent être tenus responsables d'avoir pratiqué une certaine instrumentalisation de l'historiographie au profit d'un mouvement dont ils étaient partie prenante ${ }^{26}$. Par ailleurs, pour Tournikiotis, ces mêmes auteurs, d'une part Pevsner, Emil Kauffman et Giedion et d'autre part Zevi et Leonardo Benevolo, ont utilisé l'histoire, dans une démarche qu'il qualifie d'opérative ${ }^{27}$, pour poser les fondations du Mouvement moderne et ont participé à la dénomination du courant principal de l'architecture dans les années 1920 et 1930 sous une expression unique mais recouvrant pourtant des réalités multiples. En effet, largement remis en cause depuis les années 1970, la catégorisation d'une pluralité de formes et de discours en un seul mouvement historique sous le terme de "Mouvement moderne » ou de " modernisme " apparaît comme le résultat d'une série de publications fondatrices pour la discipline, parmi lesquelles celles des auteurs cités plus haut, qui ont opéré une synthèse toute subjective. L'expression a en effet pu embrasser des "notions tout à fait distinctes : depuis celle d'un ensemble, aux frontières toujours disputées, d'édifices et de projets, à celle d'un corps de doctrine, dont les limites ne sont pas plus facile à tracer ", comme d'autre part, " recouvrir l'adhésion à un même "projet moderne" [...] projet désormais mis en doute dans son unicité même ${ }^{28}$. Considérant généralement l'ouvrage de Nikolaus Pevsner Pionners of the Modern Mouvement from William Morris to Walter Gropius comme la première occurrence de l'expression, l'historiographie semble négliger à ce propos les ouvrages propagandistes publiés par les architectes dès les années 1925 et le rôle non négligeable joué par les revues telles que L'Architecture d'Aujourd'hui créée en 1930 et L'architecture vivante

23 Panayotis Tournikiotis, The historiography of Modern Architecture, Cambridge, MIT Press, 1999 (publication de la thèse éponyme soutenue à Paris 8 sous la direction de Françoise Choay en 1988).

24 Concept développé par Tournikiotis, voir chapitre 1, "The Art Historians and the Founding Genealogies of Modern Architecture ", The historiography of Modern Architecture, op. cit.

25 Ibid.

26 Andrew Leach, What is architectural history?, op. cit.

27 Panayotis Tournikiotis, The historiography of Modern Architecture, op. cit., p. 14.

28 Hélène Jannière, "Les revues françaises de l'entre-deux-guerres et la genèse de la notion de Mouvement moderne ", dans La réception de l'architecture du mouvement moderne : image, usage, héritage, Saint-Étienne, Publications de l'Université de Saint-Étienne, 2005. 
dont Hélène Jannière explique qu'elles "sont réputées avoir été initialement fondées en vue d'apporter un soutien au "Mouvement moderne" "29. Leur rôle premier de "vecteurs de diffusion»" ${ }^{30}$, qui en fait l'objet de prédilection des historiens de la nouveauté, s'associe à une seconde vertu, contribuant au récit partisan, celle du "pouvoir de consécration symbolique ${ }^{31}$. Ainsi bien que les revues de l'époque constituèrent une grande avancée pour la diffusion de la connaissance architecturale en permettant aux architectes de connaître le travail de leurs confrères brésiliens, américains ou encore finnois et en leur donnant la parole, elles ont néanmoins participé à l'écriture d'une histoire partisane. Notamment parce qu'elles ont été créées puis dirigées par des architectes, soucieux de défendre les mouvances auxquelles ils croyaient ; mais surtout, parce qu'elles ont longtemps constitué les uniques sources de l'histoire de l'architecture.

Ayant publiés entre les années 1950 et 1960 et par-là, désignés comme les historiens de la deuxième génération, Reyner Banham, Manfredo Tafuri ou Robert Venturi ont réagi à l'identification aux valeurs du modernisme des historiens de la première génération. Au moment où le Mouvement moderne semblait montrer ses premières faiblesses, idéologiques et esthétiques et où le postmodernisme vantait les mérites d'une approche critique et sémiotique, ces historiens souhaitaient s'émanciper d'une histoire de la forme et de la fonction pour atteindre celles de l'historicité et du sens. Voyant dans cette modalité d'écriture, une erreur méthodologique, peut-être déontologique, ils ont procédé à une réévaluation critique, terme qui accompagne d'ailleurs souvent ces nouvelles histoires de l'architecture. Dans Théories et histoire de l'architecture $^{32}$, un texte dans lequel Tafuri introduit l'aspect idéologique de l'histoire de l'architecture, ce dernier reproche à Bruno Zevi, de promouvoir une méthode dans laquelle l'historien adopte et traduit les visions de l'architecte, les faisant alors tous apparaître comme complices du progrès. Tafuri remet en cause une écriture de l'histoire qui confondrait historien et architecte, ou qui ferait de l'architecte un historien, comme ce fut justement le cas de Zevi qui considérait l'histoire de l'architecture comme un versant de l'architecture, voire comme une modalité de l'architecture. À ces accusations de Tafuri à l'égard de la collaboration de certains architectes envers le Mouvement moderne s'est ajoutée plus tard une attaque contre l'architecture complice du capitalisme. Pour Jacques Guillerme, historien du projet

29 Ibid. Il faudrait peut-être nuancer cette affirmation, qui est, du reste, tirée d'un important article sur les revues d'architecture qui précise comment ces dernières, et notamment $\mathrm{AA}$, ont participé à faire émerger la notion de Mouvement moderne, mais, sans négliger d'autres approches et thématiques.

31 Ibid.

32 Manfredo Tafuri, Théories et histoire de l'architecture, Paris, Éditions SADG, 1976. 
d'architecture, "l'histoire de l'architecture [...] est plus que d'autres [sciences] sujette au détournement idéologique (un pléonasme !), spécialement l'histoire immédiate, qui fournit de curieux cas de retournements ${ }^{33}$. Reprenant Ada Huxtable dans son article "After Modern Architecture " ${ }^{34}$ qui analyse le changement selon lequel « le délai normal entre l'impulsion du projet, l'enregistrement du processus et son analyse se trouve désormais raccourci et même renversé " ${ }^{35}$, Guillerme souligne les écueils et les obstacles qui parcourent le travail d'un historien qui cherche à faire l'histoire de son temps. Il en plaisante même en évoquant des "rétrospectives d'architectes en vogue [qui] précèdent souvent leur crise de maturité ${ }^{36}$. Ainsi, ces erreurs - si tant est qu'on puisse les considérer comme telles sans abuser d'une opportunité de réfléchir sur les modalités de construction et d'écriture de l'histoire de l'architecture - invitent à reconsidérer notre travail et nos méthodologies avec le regard critique que les recherches de l'historiographie du XX $X^{\mathrm{e}}$ siècle nous ont offert en héritage.

\section{L'apport des archives de l'architecture : vers une méthodologie adaptée et renouvelée}

Partant de l'exposition des erreurs, potentielles ou réelles, qui se présentent à qui prétend faire l'histoire de l'architecture, peut-on alors imaginer une méthodologie rigoureuse, évolutive et objective ? Si les premières lignes de cet article cherchaient à mettre en application le principe selon lequel on apprend des erreurs des autres, les prochaines entendent prévenir et anticiper les erreurs que la recherche en histoire de l'architecture peut occasionner. Ces explicitations méthodologiques seront développées au regard des relations qui ont fait se rencontrer histoire de l'architecture et archives de l'architecture, à partir de leur redécouverte française. Il faut, en effet, revenir sur cette période, qui marque les années 1960-1970, pendant laquelle la manière de faire l'histoire de l'architecture s'est construite, ou plutôt reconstruite au moyen d'une exploration de ses propres sources. Alors que l'histoire de l'architecture devenait critique et s'inspirait des méthodes de l'histoire dominée à cette période par la Nouvelle Histoire, la sociologie, l'anthropologie et la « microhistoire ${ }^{37}$, un petit groupe d'acteurs du milieu de l'architecture ont mené une

33 Jacques Guillerme, «Détournements, contournements, retournements de l'histoire de l'architecture ", La recherche en architecture : un bilan international - Actes du colloque Rencontres, recherche, architecture, Marseille, Nancy, Nantes, Paris, 12-13-14 juin 1984, Marseille, Éditions Parenthèses, 1986, p. 79.

34 Ada Huxtable, "After modern architecture ", The New York Review of Books, 8 décembre 1983.

35 Jacques Guillerme, «Détournements, contournements, retournements de l'histoire de l'architecture ", op. cit., p. 79.

36 Ibid.

37 Il faut ici souligner l'influence de Carlo Ginzburg et son travail sur les sources et leur interprétation dans le cadre notamment de procès qu'il relate dans divers ouvrages. À partir de ses 
campagne en faveur de la réhabilitation de la place des archives de l'architecture au sein de la discipline. En 1975, lorsqu'André Chastel s'exprime dans la Revue de l'art pour exposer la nécessité de procéder à la conservation de ces archives, il explique que c'est uniquement grâce à l'apport de la multiplicité des documents produits dans le cadre d'un projet d'architecture que l'on pourra, au-delà de l'architecture construite, "témoigner de la recherche et de la conception $»^{38}$. Il regrette que "rien n'ait été prévu en France pour encourager le dépôt des fonds de cabinets d'architectes dans les collections publiques » et que " l'intérêt de ces archives [...] pour l'analyse d'un processus de création architecturale n'ait été compris par personne $»^{39}$. Dans une longue diatribe, il déplore la "désaffection pour le dessin d'architecture » qu'il associe à la "négligence ", la "paralysie » et l'" inaction " ${ }^{40}$ des institutions françaises.

À la suite de Chastel et des historiens de l'art, les architectes se mobilisent autour de la figure de Bernard Huet. Ce dernier, proche de Bruno Foucart ${ }^{41}$, va élever la voix dans les pages de L'Architecture d'Aujourd'hui $i^{42}$ pour appeler à la mobilisation de ses confrères en matière de protection du patrimoine. Bien qu'historiens et architectes soient partis en ordre de bataille dispersé, ils se fédéreront cependant autour de requêtes partagées : obtenir des outils de protection et des directives de la part des archives publiques. La défense du rôle des archives en histoire de l'architecture fut un succès doublement couronné par la création du Centre d'archives d'architecture de l'IFA et d'une véritable politique publique en matière d'archives d'architecture. Selon Maurice Culot, la " finalité " d'un tel lieu est "du côté [...] de la diffusion des connaissances " ${ }^{43}$. Mais elles doivent également participer à ouvrir l'amplitude du champ de conservation et de valorisation de l'architecture au-delà des avant-gardes, du Mouvement Moderne et " prendre en considération l'ensemble de l'effort architectural dans ses diversités d'écoles, de styles, de régions et de personnalités ${ }^{44}$. Voilà qui nous ramène aux discussions concernant la domination de l'histoire de l'architecture par le récit de la modernité pendant une grande moitié du XXe siècle. Maurice Culot explique qu'en conservant les archives

observations, il tire les leçons des erreurs d'interprétation possibles dans la recherche de la vérité et construit une nouvelle méthode reposant sur l'exhaustivité des sources et la réduction des échelles : la micro-histoire.

38 André Chastel, «Où sont les archives de l'architecture moderne ?», op. cit., p. 5.

39 Ibid.

40 Ibid.

41 Bruno Foucart publie, à son tour, un article sur ce sujet l'année suivante : Bruno Foucart, « La mémoire oubliée ", op. cit.

42 Bernard Huet, "Plaidoyer pour un musée d'architecture moderne ", L'architecture d'aujourd'hui, 1976, p. 5.

43 Maurice Culot, "Des archives, des chercheurs, du public, la vie!", Colonnes, n 5, juin 1992, p. 1.

44 Ibid. 
de l'architecture, il a souhaité réparer l'erreur commise par une discipline qui s'est concentrée sur un aspect, d'après lui minoritaire dans ses productions mais majoritaire par sa présence médiatique ${ }^{45}$. Et d'affirmer que «les archives d'architecture constituent la base stratégique de cette action de valorisation ${ }^{46}$.

Ainsi, la revalorisation des archives a-t-elle donné lieu à l'écriture de nouveaux récits en histoire de l'architecture, faisant la part belle à des versants longtemps négligés de l'architecture : programmes peu étudiés (architecture édilitaire, scolaire, populaire), périodes méconnues (seconde moitié du $\mathrm{XX}^{\mathrm{e}}$ siècle et $\mathrm{XIX}^{\mathrm{e}}$ siècle ${ }^{47}$ ), courants et approches mineures (régionalisme, architecture vernaculaire, balnéaire ou thermale)... Par ailleurs, la mise en œuvre d'une approche exhaustive reposant sur l'analyse de fonds d'architectes complets ou bien documentés a favorisé les démarches analytiques et détaillées aux dépens des ouvrages de synthèse, macro-historiques et généraux qui ne suscitent plus grand enthousiasme depuis les années 1980. Dans la lignée de la micro-histoire, les titres de publications et d'expositions ont porté sur des thématiques circonscrites que l'on savait pouvoir documenter comme des portraits de villes ou d'architectes de moins grande notoriété. Ce resserrement de la méthode, qui réduit l'échelle d'observation, sur des sujets précis se cristallise dans la faveur accordée, toujours d'actualité, à la monographie, qu'il s'agisse de celle d'un architecte ou d'un bâtiment ${ }^{48}$. Les fonds du Centre d'archives d'architecture de l'IFA firent l'objet dès son ouverture au public en 1991 d'études monographiques, souvent réalisées dans le cadre de thèses par des étudiants auxquels on accordait le statut de chercheur associé. On peut citer ici les travaux de Jean-Baptiste Minnaert sur Henri Sauvage ${ }^{49}$ ou de Simon Texier sur Georges-Henri Pingusson ${ }^{50}$. Quelques décennies plus tard, le bilan de cette vaste procédure de réhabilitation du document archivistique

45 Voir, par exemple, Maurice Culot, "Les archives de l'architecture moderne et contemporaine ", in Frédéric Seitz, Architecture et métal en France XIX et XXe siècles, Paris, Éditions de l'EHESS, 1994.

46 Ibid.

47 La collecte des archives relatives à cette période et la valorisation de ses productions n'est pas le fait de Maurice Culot qui, avant même de quitter l'IFA à la fin des années 1990, avait rejeté ces architectures qu'il ne jugeait pas dignes d'un intérêt suffisant pour les intégrer à la culture architecturale qu'il avait participé à faire émerger. En revanche, David Peyceré, conservateur qui officie en tant que responsable du Centre depuis 1995 a entamé la collecte des archives de l'après-guerre et des Trente Glorieuses dès les années 2000.

48 Cette thématique fit d'ailleurs l'objet d'une journée d'étude organisée par le laboratoire InTRu de l'Université François Rabelais de Tours et l'École Nationale Supérieure d'architecture de Strasbourg en juin 2013, intitulée " Microhistoire et monographie ».

49 La thèse de Jean-Baptiste Minnaert avait pour sujet : Henri Sauvage, architecte (1873-1932), thèse sous la direction de Bruno Foucart et soutenue à l'Université Paris-Sorbonne en 1993.

50 Celle de Simon Texier traitait de Georges-Henri Pingusson, architecte (1894-1978). L'architecture comme "transcendance poétique du concret " ou l'impossible doctrine, thèse sous la direction de Bruno Foucart et soutenue à l'Université Paris-Sorbonne en 1998. 
dans la recherche en histoire de l'architecture reste élogieux. Andrew Leach explique, dans un chapitre intitulé "Évidence " ${ }^{51}$, que la reconnaissance des qualités multiples des sources utiles à l'histoire de l'architecture " fut une des batailles durement gagnées des dernières décennies du XXe siècle ${ }^{52}$. De la même manière, Frédéric Seitz, qui a nourri un travail de thèse sur l'architecture métallique, considère que la conservation et la mise à disposition des archives a permis que de " travaux nouveaux [puissent] être envisagés à partir des fonds d'archives ainsi regroupés, permettant soit de renforcer des enquêtes en cours, soit de lancer de nouvelles enquêtes et, en tout état de cause, de renouveler totalement la recherche sur l'histoire économique, sociale, technique, artistique de l'art de bâtir en France " $"$.

Bien sûr, il n'y a pas de méthode infaillible. Car la recherche en histoire de l'architecture, aussi scientifique soit-elle, ne saurait considérer un canevas unique et un cahier des charges strict. Dans une démarche réflexive, elle se doit de garder à l'esprit son appartenance à un champ, celui de l'histoire de l'art, qui la prédétermine. Mais qu'est-ce-que la recherche en histoire de l'architecture ? Peut-être vaut-il mieux la définir par ce qu'elle n'est pas, par ce dont elle differe mais qu'on lui associe souvent. Ainsi, recherche en histoire de l'architecture et recherche architecturale se côtoient et se rencontrent mais ne sont pas synonymes. L'une est rétrospective, traite de l'histoire, obéit aux méthodes de la recherche scientifique et propose l'analyse et le récit. L'autre tient de la prospective, de la projection, du diagnostic et des préconisations et est souvent associée à l'innovation ${ }^{54}$. La recherche, dénominateur commun, opère selon une méthode qui, partagée par les deux directions de l'histoire et de l'innovation, interroge l'architecture par l'entrée du projet. Si elles diffèrent dans leur intention, elles sont dominées par le même objet d'étude ${ }^{55}$. Cette conception s'inspire de Gaston Bachelard et de la philosophie des sciences, qui considèrent que rien n'est donné (objet), tout est construit (projet $)^{56}$. Ce projet

51 Se traduit par " preuve ", « indice " ou « source " selon les contextes. Andrew Leach, What is architectural history?, op. cit.

52 Andrew Leach, What is architectural history?, op. cit., p. 76-96. Traduction de l'auteur de l'article.

53 Frédéric Seitz, "Les archives de l'architecture moderne et contemporaine ", Les Cabiers $d u$ Centre de Recherches Historiques. Archives, octobre 1991. En ligne : http://ccrh.revues.org/2835.

54 Cette dichotomie entre les méthodes rétrospectives et celles prospectives a été développée par Jean-Louis Chupin dans une intervention au Collège de France dans le cadre d'une journée organisée par Jean-Louis Cohen sur le thème "L'architecture entre pratique et connaissance scientifique " le 16 janvier 2015 : http://www.college-de-france.fr/site/jean-louis-cohen/ symposium-2014-2015.htm.

55 Cette rencontre autour du projet fut très précisément expliquée par Philippe Boudon lors d'une intervention au colloque Rencontres, recherche, architecture (Philippe Boudon, "L'objet de la recherche ", La recherche en architecture : un bilan international, op. cit.).

56 Jean-Louis Le Moigne, "Recherche scientifique en architecture ? ", La recherche en architecture: un bilan international, op. cit., p. 97. 
qui consiste à penser que la science est en perpétuelle reconstruction connaît son dérivé dans le champ architectural. Ainsi, le projet d'architecture ${ }^{57}$ permet d'appréhender la production dans son intégralité depuis la conception jusqu'à la réception. Cette caractérisation est également celle adoptée par les centres de conservation des fonds d'archives d'architecture qui organisent la production d'un architecte par projets plutôt que par réalisations ce qui évacuerait les projets non-construits. La prise en compte de ces modes de classification concoure à la mise en œuvre d'une méthodologie éclairée et efficiente dont le principe repose sur une bonne connaissance des modalités de production de l'architecture. Dans cette perspective, il est également important de dominer les différentes étapes qui constituent le projet ainsi que ses acteurs. À ce sujet, dans une tentative de préconisation à laquelle nous souscrirons aujourd'hui, Chastel invite à considérer " chaque étape de la construction " car " d'importants dossiers y sont fournis, qui constituent autant de pièces d'archives, de témoignages dont la disparition lèse le travail de l'historien ${ }^{58}$ ". Il poursuit ainsi, " en fait, c'est tout le circuit des papiers des architectes qu'il faut reconstituer pour retrouver, aux différentes étapes, ce qui peut être retrouvé "59. Il insiste sur la double provenance de ces documents, particularité qu'il est nécessaire d'avoir à l'esprit au début d'une recherche sur un projet d'architecture : " les deux grands axes d'acheminement sont les administrations responsables du respect de la législation en matière de construction et le client " ${ }^{60}$, auxquels nous ajouterons les documents produits par les bureaux d'étude et les ingénieurs et, à l'évidence, l'ensemble des documents produits par l'architecte lui-même. L'omission d'un de ses acteurs peut ressembler à une erreur méthodologique qu'il est aujourd'hui aisé de contourner en rassemblant consciencieusement les archives privées d'un côté (archives de la maîtrise d'œuvre et archives des bureaux d'étude et d'ingénieurs) et les archives publiques d'un autre (archives administratives pour la législation appliquée). La difficulté se situe du côté de la maîtrise d'ouvrage, qui peut être privée dans le cas d'un client indépendant (qu'il s'agisse d'une entreprise ou d'un particulier) ou bien publique comme c'est le cas quand l'État ou les collectivités lancent un chantier dans le cadre de la commande publique (aujourd'hui régie par la loi $\left.\mathrm{MOP}^{61}\right)$. L'erreur serait de penser que ces deux sources se recouvrent et se chevauchent, alors qu'en réalité « les archives des maîtres d'œuvres sont loin

57 L'expression est communément admise dans le champ de la pratique, de l'enseignement et de la recherche car, selon David Peyceré, elle « désigne aujourd'hui aussi bien l'action de projeter (la conception), le bâtiment construit - s'il est construit -, la réunion abstraite de l'idée et de sa traduction graphique (plans, maquettes, publications) " : David Peyceré, "La vie du projet ", La Gazette des Archives, n 190-191, 2000, p. 205.

58 André Chastel, "Où sont les archives de l'architecture moderne ?", op. cit., p. 7.

59 Ibid.

60 Ibid.

61 Loi no $85-704$ du 12 juillet 1985 sur la maitrise d'ouvrage publique. 
de faire double emploi, la recherche en histoire de l'architecture l'a largement démontré, avec celles de la maitrise d'ouvrage " $"$. Enfin, on peut compléter la méthodologie de Chastel en dépassant l'acceptation du terme archives comme un synonyme des " papiers de l'architecte " ou des documents - expression qui semble privilégier à nouveau l'écrit - pour considérer plus largement toutes les traces de la création architecturale : les maquettes, les esquisses, les plans, les coupes, les axonométries.

Ces précisions vont dans le sens des recherches actuelles qui tiennent compte de la pluralité des acteurs concernés par le projet et défendent une histoire de l'architecture qui ne serait plus uniquement celle des maitres d'œuvres. Ainsi, des recherches ont porté, par exemple, sur le rôle des ingénieurs dans la construction ${ }^{63}$. Parallèlement à la volonté de sortir d'une histoire de l'architecture par les architectes, des débats relatifs à l'analyse formelle et stylistique de l'architecture ont participé à la redéfinition des frontières de la discipline. Certains, comme l'historien Bruno Queysanne, défendent l'utilisation du relevé architectural pour faire l'histoire de l'architecture en avançant que la première source doit être le bâtiment lui-même ${ }^{64}$. Cette démarche, qui ne remet pas en cause mais relativise l'usage des archives d'architecture, critique " une approche socio-culturelle de l'architecture ${ }^{65}$ par laquelle « les œuvres elles-mêmes sont mises entre parenthèses " et propose d'" opposer une histoire architecturale de l'architecture [] où le premier document à considérer serait le bâtiment lui-même " et "où les outils de l'analyse seraient ceux de l'architecture : le dessin, le relevé, voire la projetation, sans oublier la description sensible $"{ }^{66}$. Cette approche, qui vise à reconsidérer le caractère formel de l'architecture, permet d'insister sur la nécessité de tenir compte de l'évolution des pratiques de la création architecturale, notamment dans la seconde moitié du XX $\mathrm{XX}^{\mathrm{e}}$ siècle. À cette période, la création architecturale se développe sous des formes très variées et connaît donc plusieurs supports - du dessin à l'infographie, de la maquette à la 3D - dont il faut tenir compte lors de la recherche et de l'analyse. L'approche défendue par Bruno Queysanne a donc pu paraître intéressante dans les années 1980 à

62 Cécile Souchon et David Peyceré, «Introduction », La Gazette des Archives, vol. 3-4, n 190-191, 2000, p. 185.

63 Voir la thèse de Christel Frapier, Les ingénieurs-conseils dans l'architecture en France, 1945-1975: réseaux et internationalisation du savoir technique, thèse sous la direction d'Antoine Picon et soutenue à l'Université Panthéon-Sorbonne en 2009 et celle de Gilles Bienvenu, De l'architecte voyer à l'ingénieur en chef des services techniques: les services d'architecture et d'urbanisme de la ville de Nantes au XVIII siècle thèse sous la direction de Gérard Monnier et soutenue à l'Université Panthéon-Sorbonne en 2013.

64 Bruno Queysanne, «En histoire de l'architecture, le document c'est le bâtiment », La recherche en architecture : un bilan international, op. cit.

65 Ibid., p. 46.

66 Ibid., p. 46. 
un moment où le dessin, qu'ils s'agissait de réhabiliter, était dénigré au profit du Dessin Assisté par Ordinateur et où l'architecture s'intellectualisait sous l'influence des philosophies déconstructivistes et structuralistes, mais elle apparaît aujourd'hui peut-être limitée et incomplète car elle ne permet pas d'envisager la pratique créative dans sa diversité de formes et de supports, voire dans son immatérialité.

Les limites de cette méthode nous invitent à envisager la redécouverte des archives de l'architecture comme une contribution à une histoire de l'architecture, tantôt trop formelle, tantôt trop hagiographique. Elles ont en effet contribué à faire redécouvrir des projets moins ou pas connus de certains architectes ou à valoriser l'œuvre d'architectes absents de la scène architecturale car il s'agissait toujours " des mêmes documents qui [étaient] reproduits, les mêmes édifices vus à travers les mêmes images ${ }^{67}$ ". C'est donc la participation au renouvellement disciplinaire qui a conduit certains historiens, à l'instar de Pierre Joly, à considérer « la question des archives, du recours aux textes, aux brouillons, aux dessins, aux ébauches " ${ }^{68}$ comme " essentielle " ${ }^{69}$. Pour Pierre Joly, « l'archive apparaissait bien plus encore qu'un moyen d'étude - en quelques sortes la matière même de notre histoire - dans toute son épaisseur ${ }^{70}$. C'est ce rapport intrinsèque entre histoire de l'architecture et archives qui amènent historiens et architectes à collaborer dès les premières collectes menées par l'IFA au milieu des années 1980. Actuellement, les difficultés de la recherche portent sur l'ambiguïté entre un grand foisonnement des sources et la pluralité des supports et des protagonistes, parmi lesquels il faut faire un tri. Longtemps considérés comme lacunaires et incomplètes, la quantité et le volume des sources de l'histoire de l'architecture présentent, au contraire, aujourd'hui une nouvelle difficulté tant pour le chercheur, que pour l'archiviste. Cette caractéristique s'explique par l'augmentation progressive du nombre de projets (construits ou non) et surtout de collaborateurs dans les agences, l'accumulation des documents par les producteurs (fussentils privés ou publics) et une législation française en matière de patrimoine bâti et de construction qui n'a pas été vers la simplification pourtant recherchée. Le Centre d'archives d'architecture de l'IFA s'est engagé au côté d'autres institutions internationales, comme 'ICAM $^{71}$ ou le $\mathrm{CIA}^{72}$, dans un travail de médiation et de soutien méthodologique aux institutions collectrices qui s'est

67 Pierre Joly, "Introduction ", in Sabine Kühbacher et Pierre Joly, Archives et histoire de l'architecture : actes du colloque des 5, 6 et 7 mai 1988 à Paris, Paris, éd. École d'architecture de Paris-La Villette, 1990, p. 12.

68 Ibid.

69 Ibid.

70 Ibid., p. 13.

71 International Confederation of Architectural Museums.

72 Conseil International des Archives. 
traduit par la publication d'un « Manuel de traitement des archives de l'architecture $»^{73}$ en 2000 et de deux guides faisant l'état des fonds d'architectes ${ }^{74}$ en France dans les années 1990. Au même moment, la volonté de réparer les erreurs et confusions historiographiques passées auxquelles la collecte des archives a contribué s'est étendue au champ de l'enseignement de l'histoire de l'architecture. À ce propos, les expériences menées par Richard Klein à l'École d'architecture de Lille dans son séminaire "Archéologie du projet " 75 s'inscrivent dans la refonte de l'enseignement de l'architecture après 1968 et dans la prise de conscience généralisée de l'importance du contexte historique.

Ainsi, il existe autant de modalités de l'erreur qu'il existe de manières de faire et d'écrire l'histoire de l'architecture. Plusieurs d'entre elles ont été envisagées ici, à commencer par les erreurs méthodologiques évidentes, isolées et occasionnelles : erreur d'interprétation, erreur par ignorance, erreur de datation. L'affaire se complique devant les erreurs partagées et collectives qui peuvent aller de l'erreur de répétition à l'erreur polémique ou controverse. Enfin, l'erreur peut être - et c'est là certainement la plus répandue et donc la plus complexe - militante, voire partisane. Certaines erreurs semblent ainsi s'être multipliées dans l'histoire, voire avoir fait école mais selon l'adage qui veut que l'on apprenne de ses erreurs, l'histoire de l'architecture semble en être sortie grandie. Enfin, au-delà de l'histoire, c'est la création architecturale qui a bénéficié des erreurs qui ont marqué l'histoire de l'architecture, notamment parce que leurs effets ou répercussions ont été transmis aux architectes par l'intermédiaire de l'enseignement qu'ils ont reçu, par leur rencontres avec l'histoire lors de rénovations et de réhabilitations ou tout simplement par conscience de l'héritage qui leur est légué.

L'erreur en histoire de l'architecture revêt donc bien des aspects qui invitent à la considérer moins comme une action à regretter ou à réparer que comme une démarche constructrice, voire fondatrice d'une discipline et de ses méthodes. En reprenant les termes de Gaston Bachelard qui estimait que "l'esprit scientifique se constitue sur un ensemble d'erreurs rectifiées " ${ }^{76}$,

73 Conseil international des archives et Section des archives d'architecture, op. cit.

74 Institut Français d'Architecture, Archives d'architecture du XX'e siècle, Liège, Mardaga, 1991 et David Peyceré, Gilles Ragot (éd.), Archives d'architectes : état des fonds, XIX'e-XXe siècles, Paris, La Documentation française, Institut Français d'architecture et Direction des archives de France, 1996.

75 Richard Klein, Séminaire de recherche de $3^{e}$ cycle "Archéologie du projet " tenu à l'École d'architecture de Lille.

76 Bachelard Gaston, La formation de l'esprit scientifique : contribution à une psychanalyse de la connaissance, Paris, J. Vrin, 1938. Cité par Louisa Youfsi, "L'épistémologie de Bachelard : entre ruptures et continuité, Sciences Humaines, $n^{\circ} 242$, novembre 2012, consultation en ligne http://www.scienceshumaines.com/l-epistemologie-de-bachelard-entre-ruptures-et-discontinuite_fr_29573.html. 
il apparaît séduisant de penser que la mise à l'épreuve, l'introspection et la rupture qu'entraîne l'erreur puissent être constitutives d'une méthodologie exigeante de la recherche, en histoire de l'architecture comme ailleurs.

\author{
Nina Mansion \\ EA 538 Centre François-Georges Pariset \\ Université Bordeaux Montaigne \\ École du Louvre Paris \\ nina.mansion@etu.u-bordeaux-montaigne.fr
}

\title{
Résumé
}

Cet article entend interroger les méthodes de l'histoire de l'architecture au regard d'un travail de définition de la notion d'" erreur " dans le champ de la recherche scientifique. Il s'agit, ensuite, d'analyser la contribution de la redécouverte et de la collecte des archives de l'architecture à partir des années 1970 en France au renouvellement méthodologique et épistémologique en histoire de l'architecture.

\section{Mots-clefs}

Archives de l'architecture, histoire de l'architecture, méthodologie de la recherche, Institut Français d'architecture, historiographie.

\section{Abstract}

In the 1970's, André Chastel and various actors of Architecture called for measures regarding the alarming situation that architectural archives and records in France are facing. The recognition and the collecting of theses architectural artefacts contribute to the renewal of the history of architecture as a discipline which is simultaneously examined with regards to the various errors implanted in its construction.

\section{Keywords}

Architectural archives and records, history of architecture, research methodology, historiography. 